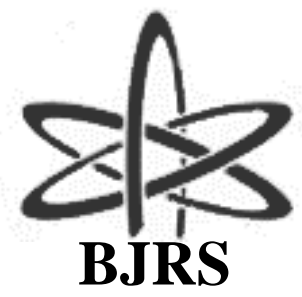

BRAZILIAN JOURNAL

$\mathrm{OF}$

RADIATION SCIENCES

08-02 (2020) 01-18

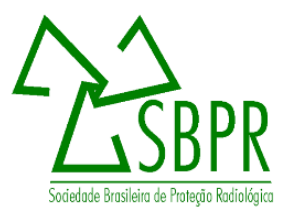

\title{
Estudo de eventos operacionais de reatores refrigerados e moderados à água leve pressurizada usando o banco de dados do IRS/IAEA (2010-2019)
}

\author{
Vital $^{\mathrm{a}}$ R.B.N., Vital ${ }^{\mathrm{b}}$ T.M. \\ ${ }^{a}$ Universidade Estácio de Sá, 23914-360, Angra dos Reis, Rio de Janeiro, Brasil \\ richardvital@gmail.com \\ ${ }^{b}$ tati.mv7@gmail.com
}

\section{RESUMO}

O conhecimento das características de eventos rotineiros em usinas nucleares permite o estabelecimento de ações para evitar a ocorrência, atendendo aos princípios de segurança nuclear cujo objetivo é proteger as pessoas e o meio ambiente dos riscos associados à atividade industrial. Nesse sentido, a existência de um mecanismo de trocas de informações entre organismos de diversas partes do mundo auxilia no processo de tomada de decisões. Com base nos princípios da cultura de segurança que propõe um aperfeiçoamento contínuo das práticas operacionais, este trabalho mostra os resultados de um levantamento realizado em 293 relatórios de eventos, registrados no IRS, de diversas usinas nucleares refrigeradas e moderadas à água leve pressurizada, segundo a codificação das suas características de acordo com o guia de referência. Dessa forma, o objetivo deste trabalho é identificar as principais características dos eventos que ocorreram no período de 2010 a 2019. Os resultados demonstram a necessidade de constante aperfeiçoamento das práticas operacionais com a priorização da cultura de segurança em detrimento das questões financeiras, além da importância que deve ser dada aos treinamentos para reduzir $o$ impacto das falhas humanas.

Palavra Chave: Experiência Operacional, Reator à Água Pressurizada, Segurança Nuclear, Relatório de Evento, Sistema para registro de eventos operacionais. 


\begin{abstract}
The knowledge of the characteristics of routine event in nuclear power plants allows the anticipation of actions to avoid the occurrence, taking in account the principles of the nuclear safety whose main objective is to protect people and environment form risks associated with this industrial activity. In this sense, the existence of mechanisms for organizations in different part of the world to exchange information can helps in the decisionmaking process. Based on the principle of safety culture that proposes a continuous improvement of operational practices, this paper shows the results of a survey of 293 event reports registered in IRS database considering several Light Water Reactors around the world, regarding the coding of main event characteristics according with the reference guide. Thus, the objective of this work is to identify the main characteristics of the events that occur between 2010 and 2019.
\end{abstract}

Keywords: Operating Experience, Pressurized Water Reactor, Nuclear Safety, Event report, Incident Reporting System. 


\section{INTRODUÇÃO}

As indústrias buscam constantemente aprimorar seus processos para fornecer soluções que possam reduzir custos e, consequentemente, aumentar o retorno econômico financeiro de suas atividades, assumindo diferentes níveis de risco de acordo com a sua área de atuação. Por outro lado, vários acidentes demonstram a importância de se criarem ferramentas e métodos para reduzir riscos industriais e suas consequências para a população ou para o meio ambiente. Baseado no conceito de implementação da cultura de segurança, o setor nuclear utiliza métodos para trocar informações entre diferentes entidades: representantes da área produtiva, organismos de regulação ou instituições de pesquisa e desenvolvimento [1]. Nesse processo, chamado de Experiência Operacional, ocorre a coleta de informações, a análise de eventos ocorridos e a avaliação da sua aplicabilidade a um projeto com características compatíveis. Dessa forma, a existência de um mecanismo de troca de experiências pode evitar a ocorrência de um evento. Além disso, o conhecimento das características dos eventos auxilia no processo de tomada de decisões [2].

O arcabouço legal para a área nuclear no Brasil é definido pela Comissão Nacional de Energia Nuclear (CNEN). A Norma CNEN 1.14 [3] define a necessidade de notificar e enviar um relatório dependendo do tipo de evento ocorrido em uma usina nuclear [4]. A Agência Internacional de Energia Atômica (IAEA) também possui um conjunto de Guias com recomendações para auxiliar e incentivar a troca de experiências sobre os eventos operacionais [5].

Atualmente, existem mais de quatrocentos reatores nucleares em operação pelo mundo [6]. Considerando os reatores em operação no ano de 2019, aproximadamente $66,1 \%$ são moderados e refrigerados à água leve pressurizada (LWR - Light Water Reactor) [7]. Esse volume de instalações permite a aquisição de uma relevante quantidade de informações para estudo. A IAEA junto à Agência de Energia Nuclear (NEA) desenvolveu um sistema para troca de experiências, International Reporting System (IRS), cujo objetivo é permitir a análise e o registro de eventos operacionais [4]. Neste sistema, os eventos das usinas de países membros são selecionados por um coordenador local e são analisados em um encontro anual para que, posteriormente, sejam disponibilizados para consulta. O coordenador de cada país deve selecionar os eventos mais relevantes e codificar as principais informações do mesmo [8]. 
A fim de avaliar as principais características de eventos em reatores moderados e refrigerados à água pressurizada compatíveis com as usinas nucleares em operação no território brasileiro, este trabalho tem como objetivo filtrar e apontar as conclusões mais importantes extraídas de 293 eventos registrados no IRS que ocorreram entre os anos de 2010 e 2019.

\section{MATERIAIS E MÉTODOS}

Os eventos incluídos no banco de dados do IRS são codificados conforme as diretrizes apresentadas no documento Manual for IRS Coding joint IAEA/NEA International Reporting System for Operating Experience Service Series $N^{\circ} 20$ [8]. Esse documento fornece orientações para codificação padronizada de eventos, através de nove grupos de códigos, que serão descritos na segunda etapa do processo ilustrado na Figura 1.

O processo de análise das informações apresentadas nos relatórios de eventos registrados no IRS consiste em três etapas como ilustrado na Figura 1.
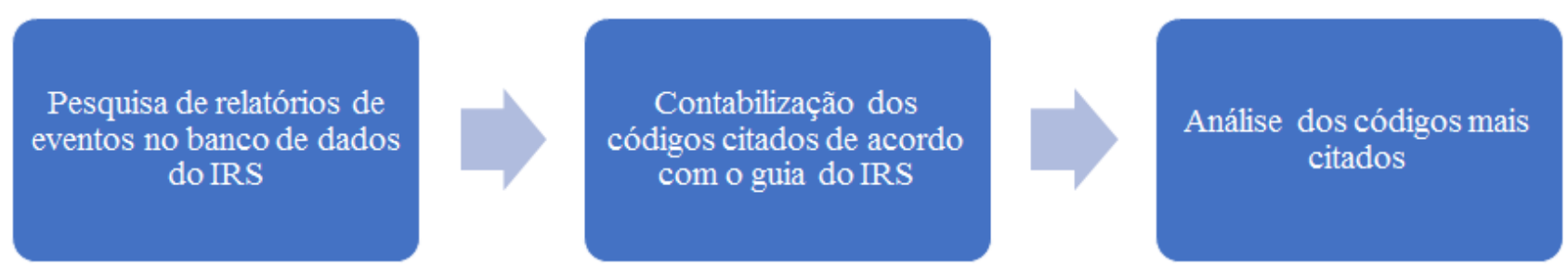

Figura 1: Etapas do estudo sobre eventos operacionais em reatores resfriado e moderados à água leve pressurizada

A primeira etapa da pesquisa foi a definição do escopo dos relatórios de eventos que seriam estudados. Durante essa etapa, extraíram-se as informações do banco de dados do IRS usando um filtro para reatores refrigerados e moderados à água leve pressurizada. Nesse grupo de reatores, encontram-se diversos modelos e fabricantes construídos sobre duas concepções: PWR (Pressurized 
Water Reactor) e VVER (Water-Water Energetic Reactor). A escolha desses modelos de reatores tem como base os projetos das usinas nucleares brasileiras que são do tipo PWR e as semelhanças com os reatores VVER. Angra 1 é um projeto PWR americano da empresa Westinghouse e Angra 2 é um projeto PWR alemão que foi desenvolvido pelas empresas Siemens e KWU [9]. Devido à quantidade de informações registradas no banco de dados, aplicou-se outro filtro na pesquisa referente ao período de ocorrência do evento e, esse foi limitado entre os anos de 2010 a 2019 para garantir uma amostra estatística representativa. O resultado final foi uma lista composta por 293 relatórios de eventos.

Na segunda etapa do estudo, agruparam-se os eventos segundo os códigos presentes no guia da IAEA que são divididos em nove grupos de informações. O primeiro grupo refere-se a algumas categorias de eventos pré-definidas (categoria de relatórios), como por exemplo, liberação de radiação, perda das barreiras de proteção, degradação ou falha em sistema de segurança, etc. $\mathrm{O}$ segundo grupo de códigos descreve a situação da planta antes da ocorrência do evento, ou seja, se a usina estava em potência, desligada para recarregamento ou por motivo de segurança, sob alguma variação de potência ou ainda, nas fases de construção ou comissionamento. No terceiro grupo, encontram-se as informações de sistemas falhados ou afetados, sendo alguns deles: refrigeração do reator (SRR); auxiliares do reator; elétricos; circuito secundário, aquecimento e refrigeração; instrumentação e controle (I\&C); dentre outros. Componentes falhados ou afetados são avaliados dentro do quarto grupo, por exemplo: transmissores de I\&C, computadores, controladores, válvulas, tubulações, bombas, trocadores de calor, disjuntores, fusíveis, relés, etc. O quinto grupo de códigos está relacionado aos resultados da análise de eventos evidenciando a causa direta e aspectos relacionados ao desempenho humano, organizacional ou de equipamento. Os efeitos dos eventos para a operação da planta são analisados no sexto grupo de códigos que contemplam consequências do tipo: desligamento do reator, variação de potência, liberação de radiação, não atendimento a limites regulados, perdas financeiras, etc. O sétimo grupo (características do evento) refere-se às ações observadas durante o transiente em investigação. Os oitavo e nono conjuntos descrevem a natureza da falha e a forma de restabelecimento da usina (ações de recuperação da usina) para manter uma condição segura.

$\mathrm{Na}$ terceira etapa, foram identificados os códigos mais citados e pesquisadas outras referências bibliográficas relacionadas ao código de evento em análise. 


\section{RESULTADOS E DISCUSSÃO}

A Figura 2 mostra a distribuição de eventos citados para o grupo "Categoria de Relatórios" que possui vinte e seis codificações disponíveis. Os dados coletados mostram que o código relacionado a "Deficiências na Operação" é o mais citado nos relatórios. Esse tipo de deficiência está relacionado a falhas humanas, deficiências ou não aderência a procedimentos, treinamento inadequado, dentre outros [8]. O segundo código mais citado é "Eventos de potencial significativo para a segurança" que engloba uma série de eventos com baixa significância, eventos recorrentes, problemas genéricos quanto ao mecanismo de troca de experiência, pesquisa ou regulação. Na terceira posição, aparecem as "Deficiências de projeto" que podem ocasionar uma perda de um sistema ou função de segurança, falhas de modo comum e aplicação de materiais incompatíveis com as condições ambientais ou operacionais.

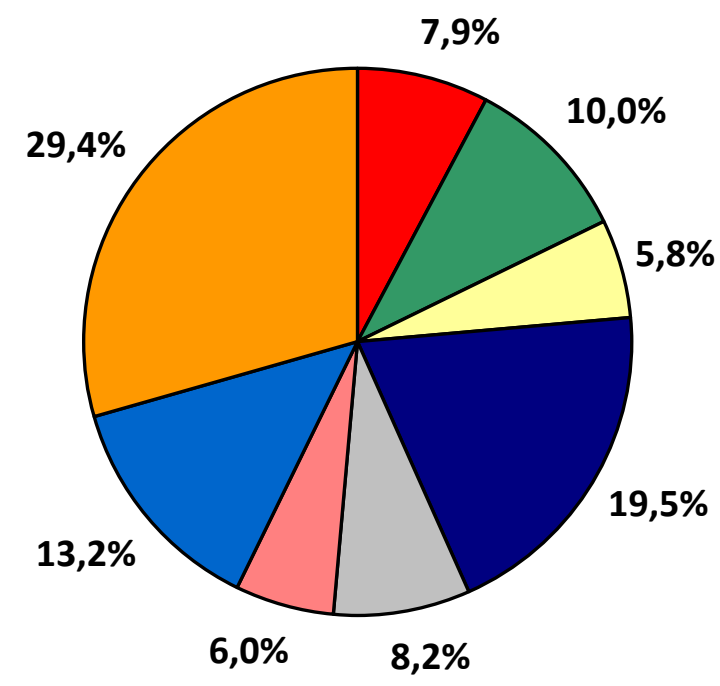

1.2.6 - Degradação de um sistema de suporte essencial

$\square$ 1.3.1 - Deficiências de projeto

$\square$ 1.3.2 - Deficiências na construção, instalação e comissionamento

1.3.3 - Deficiências na operação

$\square$ 1.3.4 - Deficiências de gerenciamento de segurança ou sistema de garantia da qualidade

$\square \mathbf{1 . 4}$ - Problema genérico de interesse para a segurança

$\square$ 1.6 - Eventos de potencial significativo para a segurança

$\square$ Soma dos outros

Figura 2: Distribuição percentual dos eventos operacionais dentro do grupo "Categoria de Relatórios"

Em um conjunto de vinte e três codificações possíveis, a Figura 3 mostra que 40,2\% dos eventos pesquisados ocorreram quando as usinas operavam em plena potência. $\mathrm{O}$ segundo grupo de 
condição operacional citado nos relatórios está relacionado a testes e manutenção. Outra condição importante observada é a de recarregamento e, na maioria dos casos, os eventos ocorrem com elementos combustíveis no núcleo do reator.

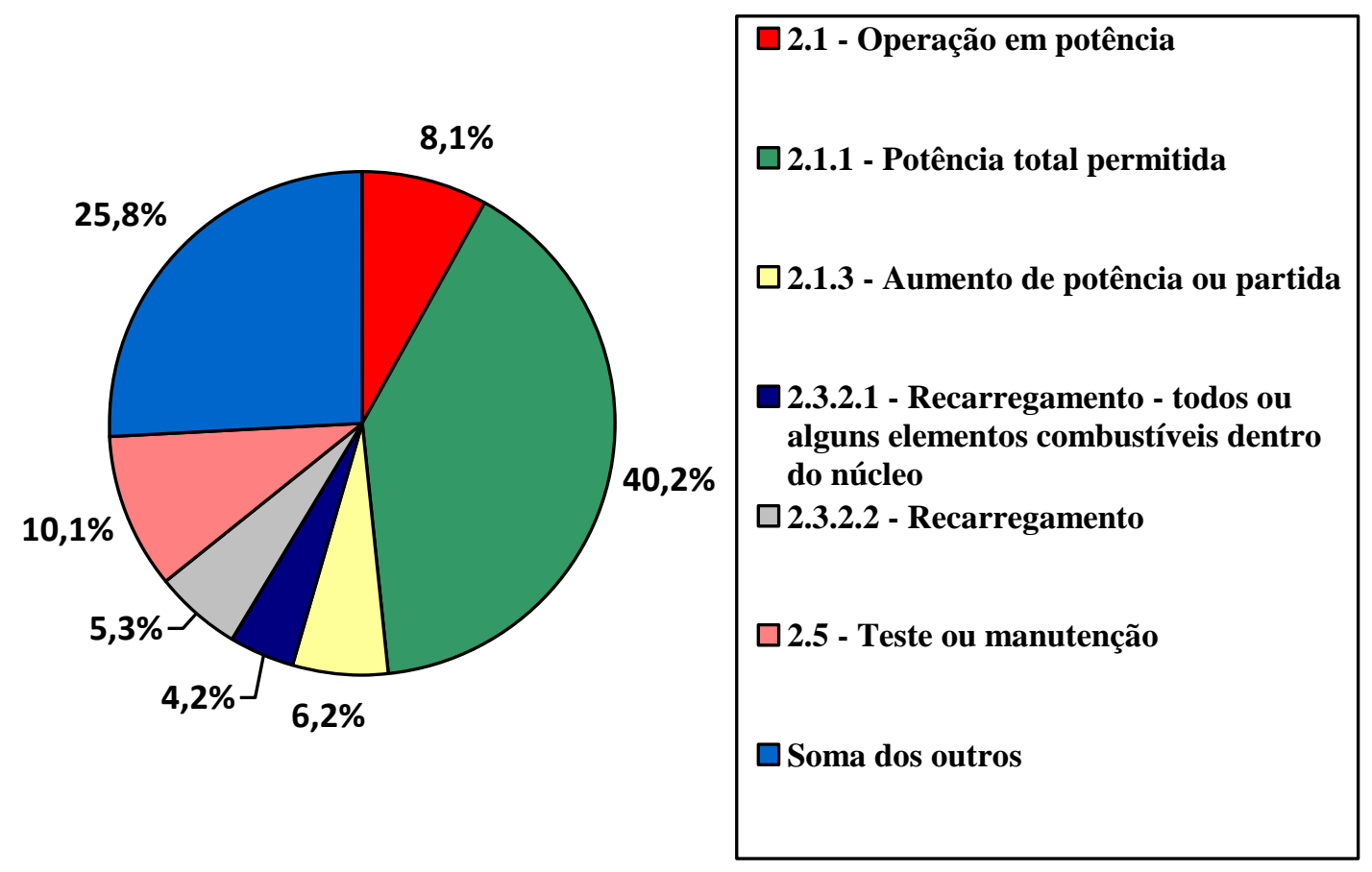

Figura 3: Ocorrência de eventos para o grupo "Situação da planta antes da ocorrência do evento"

Dos cento e vinte e quatro códigos disponíveis para "Sistemas falhados ou afetados", o código mais citado na Figura 4 está relacionado aos geradores diesel de emergência e seus sistemas associados (óleo lubrificante, óleo combustível, resfriamento das camisas de partida, etc), registrados em 6,6\% dos relatórios analisados. Apesar do percentual do código mais mencionado não representar uma grande parcela do total, verifica-se que este é significativo devido ao grande número de possibilidades. A reconhecida importância do funcionamento dos sistemas de segurança dos geradores diesel de emergência em projetos segurança das usinas levou ao desenvolvimento de um estudo para análise dos eventos relacionados a esses sistemas [10]. A segunda codificação de maior frequência está relacionada ao sistema de refrigeração do reator, registrada em 5,6\% dos relatórios. O terceiro sistema, apontado em 4,7\% dos casos, é dos Geradores de Vapor que são barreiras contra a liberação de radioatividade para o meio ambiente. 


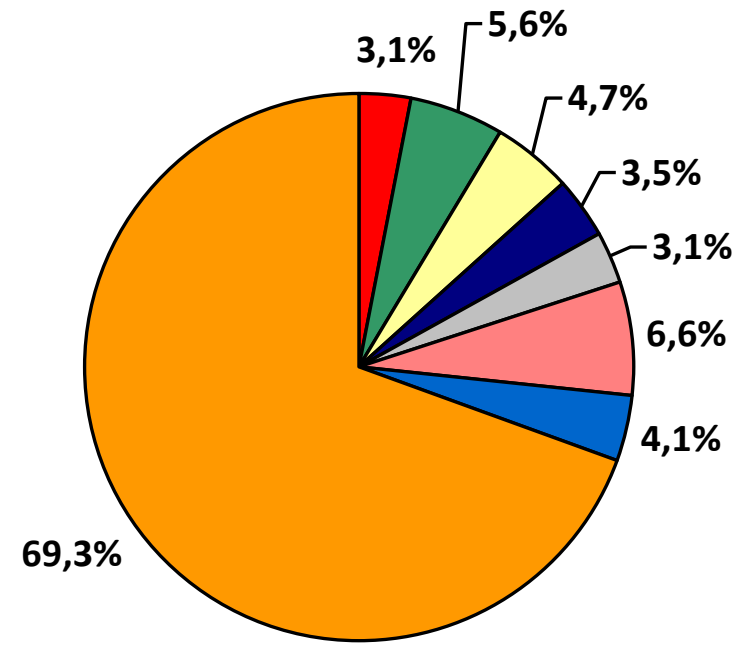

3.AB - Sistema de controle e proteção do reator

$\square$ 3.AE - Sistema de refrigeração do reator

$\square$ 3.AH - Gerador de Vapor

3.CB - Sistema de água de serviço

3.EB - Sistema de corrente alternada em média tensão

$\square$ 3.EF - Geradores de emergência e seus sistema associados

3.FG - Sistema de água de alimentação e condensado

$\square$ Soma dos outros

Figura 4: Ocorrência de eventos para o grupo "Sistema falhados ou afetados"

O grupo "Componente falhado ou afetado" é composto por vinte e seis codificações, cujo resultado do estudo é mostrado na Figura 5. Os resultados indicam que os componentes com mais citações são as válvulas e seus sistemas de acionamento que representam 15,2\% do universo analisado. Uma análise indicando que as válvulas são os componentes mais afetados no conjunto de eventos analisados é mostrada em [11]. Componentes como "Tubos e dutos" aparecem em 8,9\% dos relatórios analisados. Um estudo para as usinas PWR alemãs que relaciona a falha de componentes mecânicos como tubulações a um processo de degradação por envelhecimento é mostrado em [12]. Componentes elétricos como relés, conectores e chaves fazem parte do terceiro grupo mais mencionado, representando 7,6\% das ocorrências. Um estudo para as principais causas que levaram a falhas em componentes, recomendando práticas e reforçando a importância da utilização de informações de experiência operacional é mostrado em [13]. 


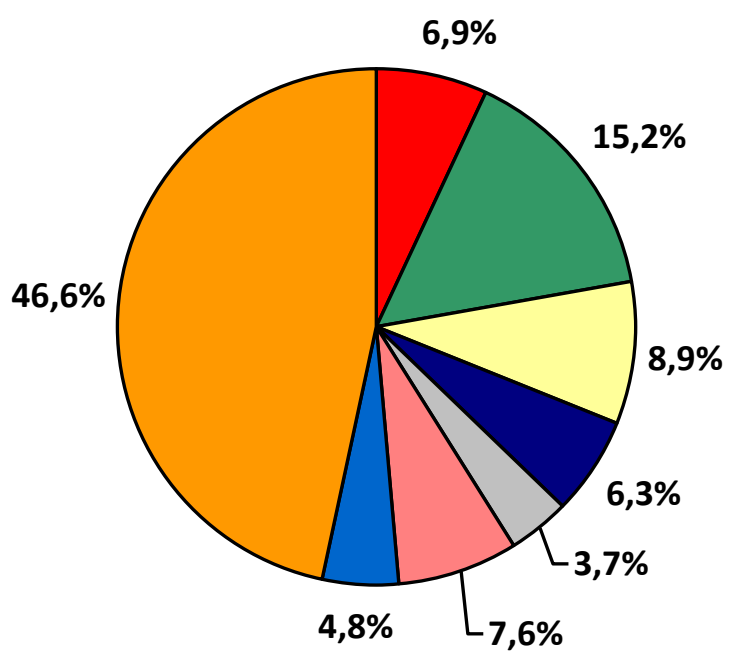

4.2.1 - Bombas e compressores

$\square$ 4.2.3 - Válvulas

$\square$ 4.2.6 - Tubos e dutos

4.3.1 - Transformadores, barramentos e isoladores de linha

$\square$ 4.3.2 - Disjuntores e fusíveis

$\square$ 4.3.7 - Relés, conectores e chaves

4.3.8 - Controladores de linha e cabos elétricos

$\square$ Soma dos outros

Figura 5: Ocorrência de eventos para o grupo "Componente falhado ou afetado"

Dois códigos foram citados igualmente para o grupo causa direta e estes são relativos à "Manutenção e testes periódicos de equipamentos" e "Envolvimento da equipe de manutenção", correspondendo a aproximadamente $2,8 \%$ de um conjunto de cento e sessenta e sete possibilidades, como pode ser observado na Figura 6. O segundo código mais recorrente relaciona o desempenho humano e a qualidade dos procedimentos. Devido à importância da atuação humana na segurança das usinas, um estudo sobre o impacto dos fatores organizacionais sobre o desempenho humano é abordado em [14]. 


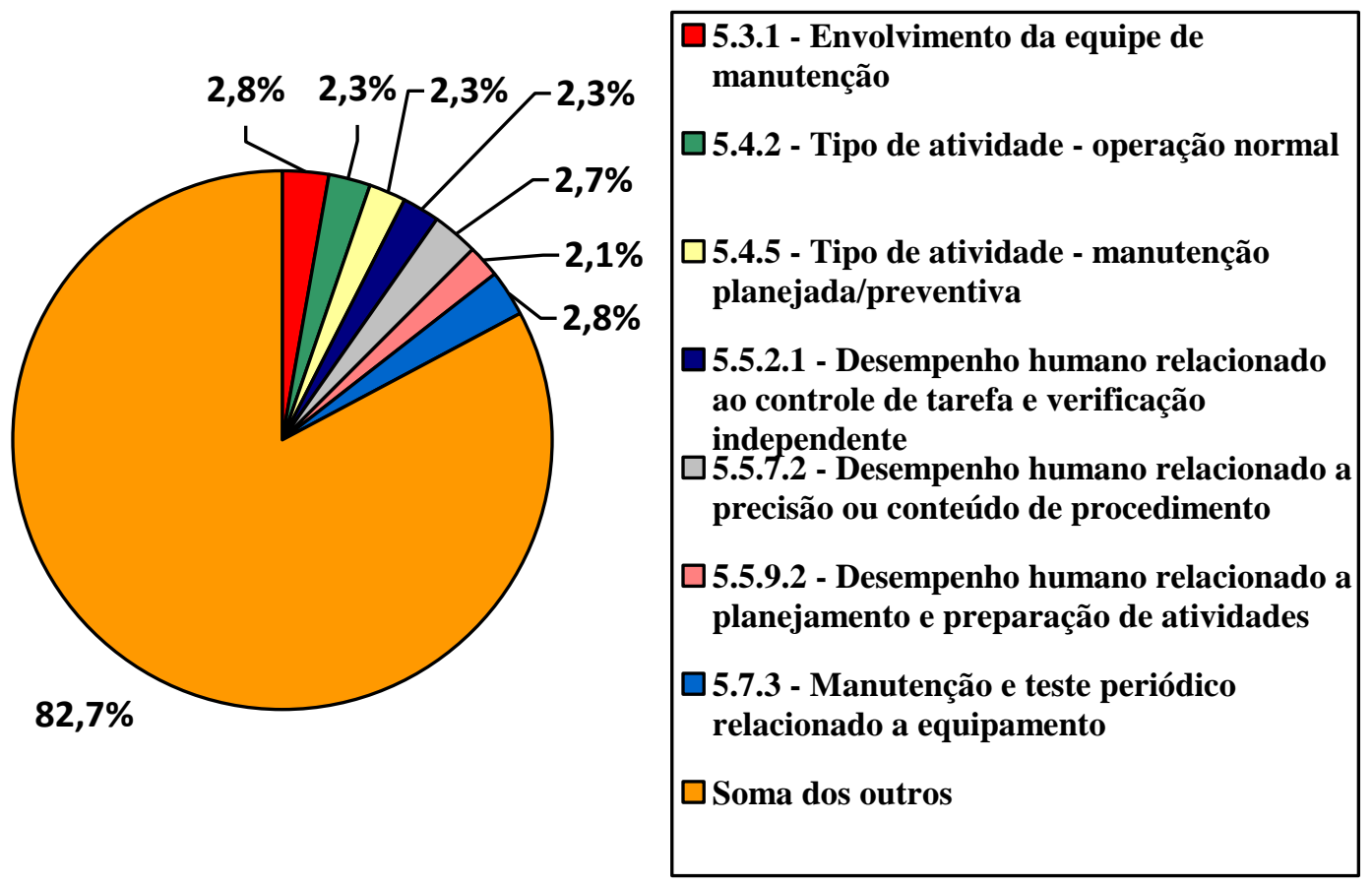

Figura 6: Ocorrência de eventos para o grupo "Causa direta"

Dentre vinte e um códigos para o grupo "Efeitos na operação", na Figura 7, verifica-se que 28,3\% dos códigos citados não apresentaram impactos relevantes para a operação, o que reforça a importância do estabelecimento de uma cultura de segurança e defesa em diversos níveis de profundidade. Desligamento automático do reator, que constitui uma importante ação preventiva para manter a planta em uma condição segura, é o segundo código mais apontado, representando 15,0 \% do total de eventos. Com 8,7\% de representação, "Desligamento controlado" e "Limites das Especificações Técnicas excedidos" dividem a terceira posição nos registros de eventos avaliados. As Especificações Técnicas contêm os limites e os requisitos para garantir a confiabilidade dos sistemas de segurança das usinas atendendo aos requisitos definidos no Relatório Final de Análise de Segurança (RFAS) [15-16]. 


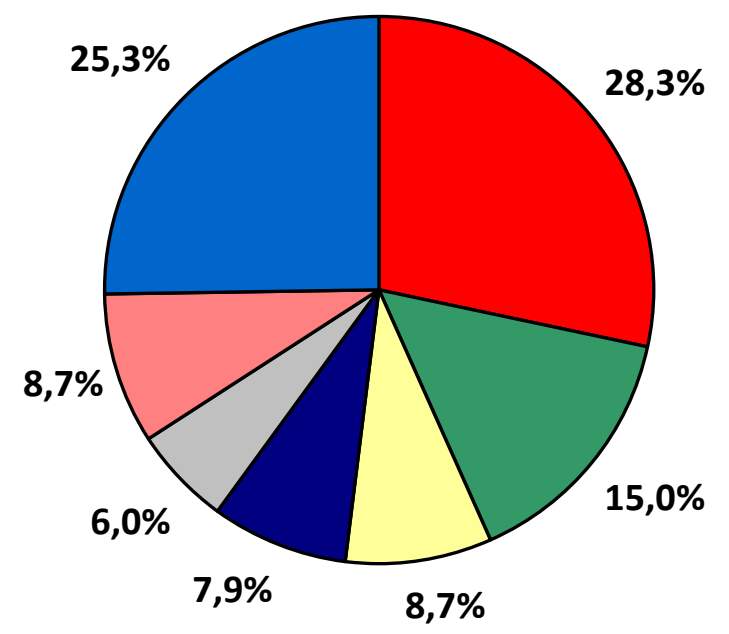

$\square 6.0$ - Nenhum efeito significante para a operação

$\square$ 6.1.1 - Desligamento automático do reator

$\square 6.2$ - Desligamento controlado

6.4 - Acionamento de recurso de segurança

$\square 6.9$ - Extensão da parada para recarregamento

$\square 6.10$ - Limites das Especificações Técnicas excedidos

$\square$ Soma dos outros

Figura 7: Ocorrência de eventos para o grupo "Efeitos na operação"

Ao se analisar a Figura 8, verifica-se que o código mais citado para as "Características do evento" indica uma condição que não está coberta pelos códigos existentes. Na segunda colocação, aparece a codificação relacionada às oscilações de potência, com ocorrência de 11,9\%. Variações da potência nuclear e suas consequências para a segurança de uma usina são mostradas em [17]. Devido à importância do controle de potência no núcleo em um reator, falhas com essas caraterísticas são consideradas no relatório de análise de segurança da usina [15]. Possíveis degradações de uma função de segurança ocorreram em 10,4\% das citações. A implementação das funções críticas de segurança no processo de licenciamento de usinas nucleares é resultado da aplicação dos conceitos de experiência operacional durante a análise do evento ocorrido na usina americana de Three Miles Island [18]. 


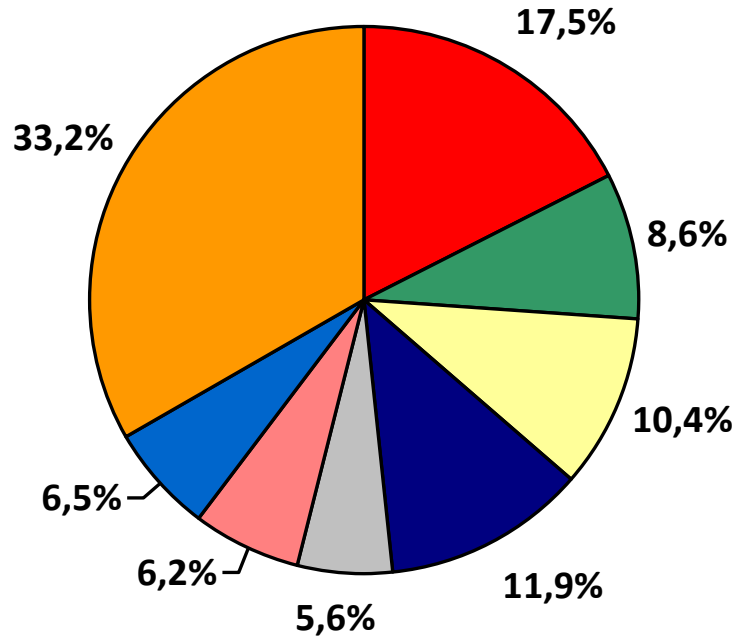

$\square 7.0$ - Outras características

$\square 7.2$ - Degradação no limite de refrigeração do reator

$\square 7.5$ - Degradação significativa de uma função de segurança

7.11.1 - Transiente de potência

$\square 7.11 .3$ - Transiente de pressão

$\square 7.12$ - Perigo físico

7.13 - Descoberta de uma condição não considerada ou analisada

$\square$ Soma dos outros

Figura 8: Ocorrência de eventos para o grupo "Características do evento"

Dentre os oito códigos disponíveis para o grupo "Natureza das falhas ou erros", a Figura 9 mostra que o mais comum é o de falhas ou erros simples com $35,7 \%$ das citações. As falhas ou erros múltiplos em sistemas independentes representam 17,0\% das classificações, o que demonstra a importância do emprego de técnicas de redundância e diversidade nos projetos de reatores [19]. As falhas de causa comum representam $13,2 \%$ do total de menções. Esse tipo de falha causa preocupação por reduzir a confiabilidade dos sistemas de segurança [20]. 


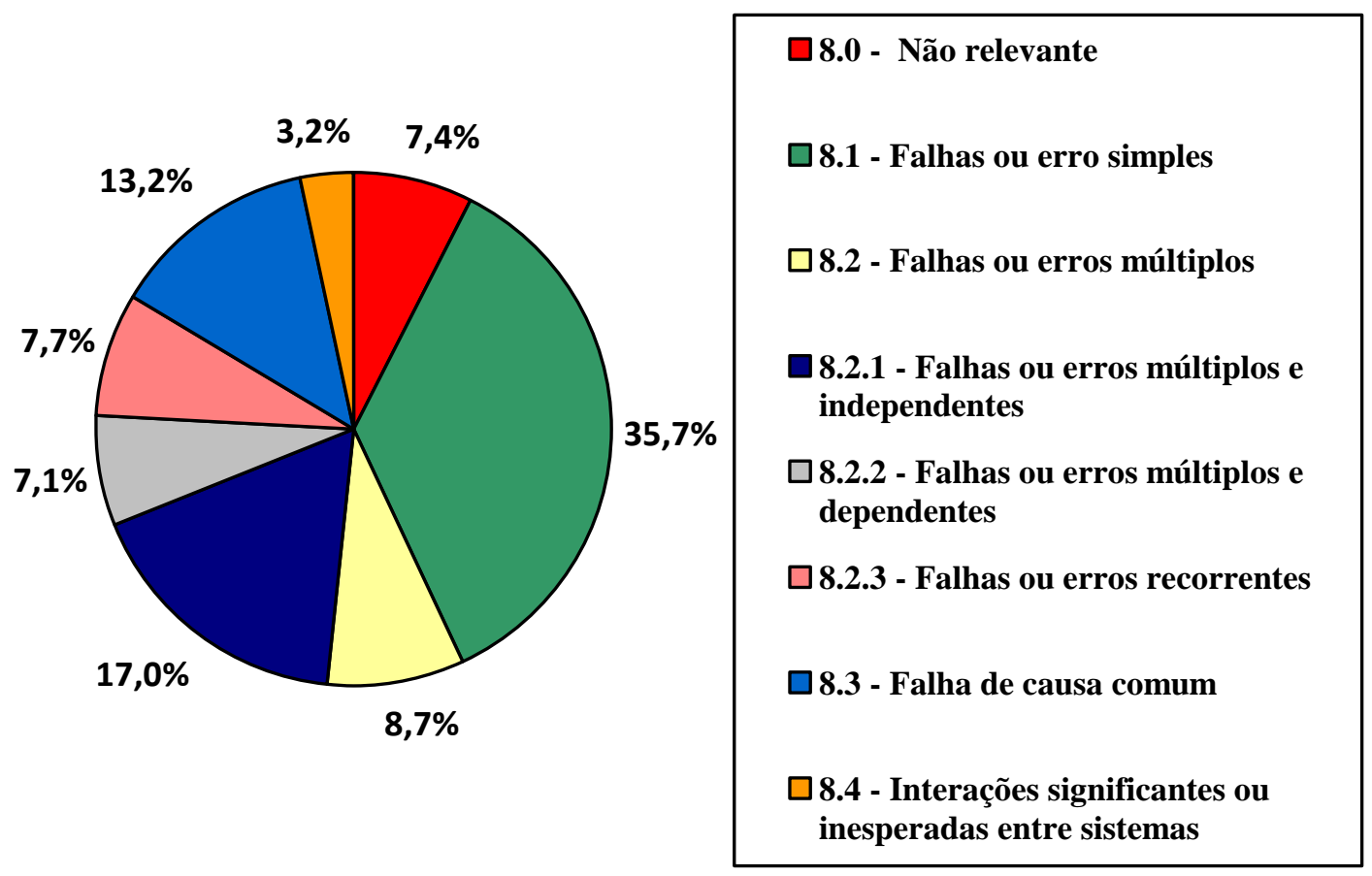

Figura 9: Caracterização de eventos do grupo "Natureza das falhas ou erros"

A Figura 10 mostra que a recuperação das condições das usinas ocorreu em 50,7\% devido à ação humana seguindo rotinas e procedimentos previstos. Os dados apresentados também demonstram a importância dos sistemas automáticos da usina para a recuperação da mesma, visto que $10,3 \%$ das citações ocorreram sem atuação humana. 


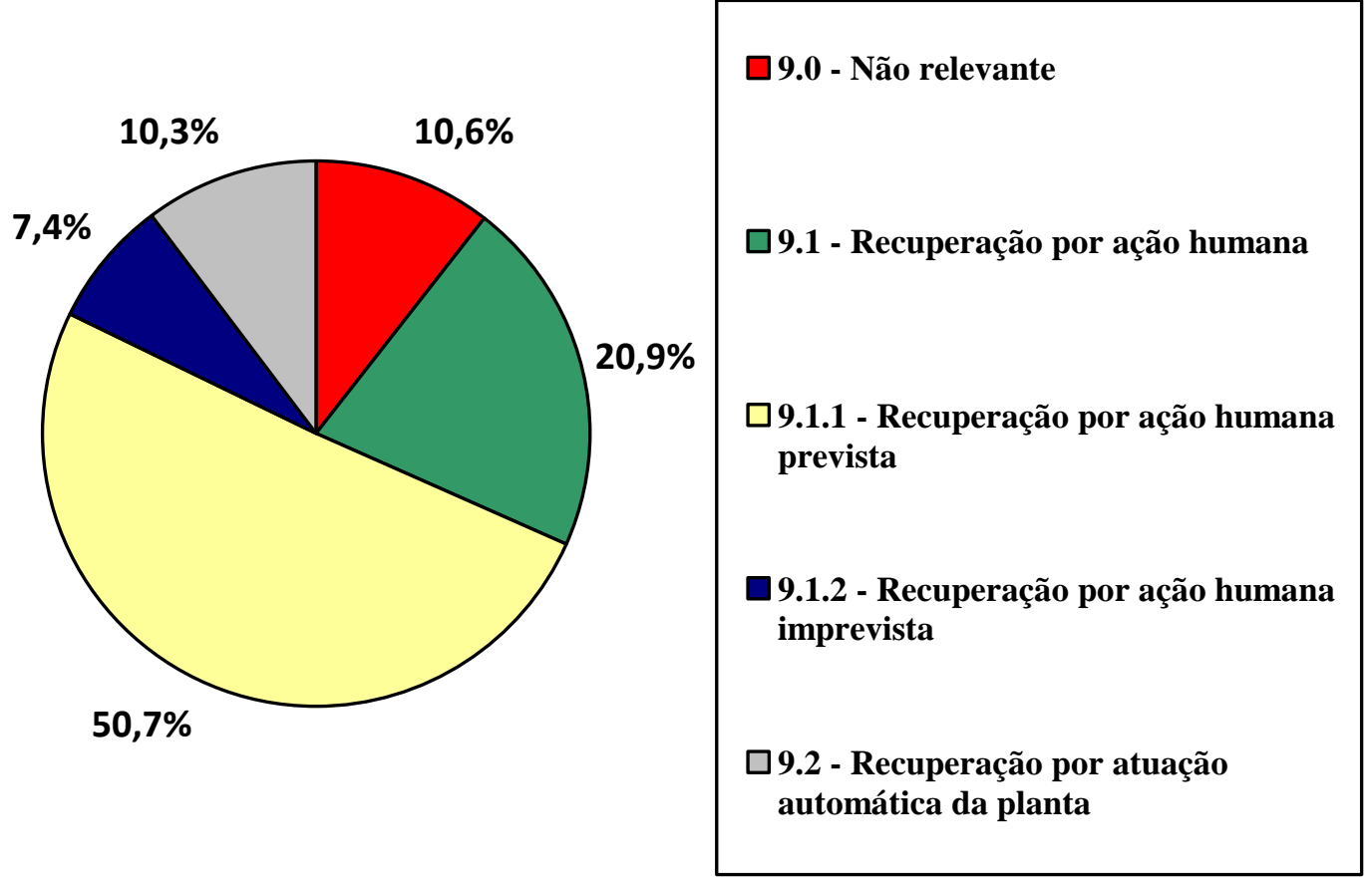

Figura 10: Distribuição dos eventos para o grupo "Ações de recuperação da usina"

\section{CONCLUSÃO}

Este trabalho reforça a importância da área de Experiência Operacional para a segurança de usinas nucleares e, mostra como as ferramentas de armazenamento e análise auxiliam na prevenção da recorrência de eventos. O banco de dados do IRS tem informações suficientes para auxiliar o aperfeiçoamento de variados programas (manutenção, desempenho humano, controle de configuração, gerenciamento de envelhecimento, entre outros) em diversos tipos de reatores refrigerados e moderados à água leve pressurizada. Os erros pontuais no processo de seleção dos eventos ou codificação realizada não são relevantes quando se analisa uma grande amostra de eventos.

Os resultados demonstram que para a "Categoria dos Relatórios", o somatório dos quatro códigos mais citados (deficiências na operação; eventos de potencial significativo para a segurança; deficiências de projeto; deficiências de gerenciamento de segurança ou sistema de garantia da qualidade) supera a metade dos casos registrados, demonstrando que as usinas precisam destinar 
atenção especial a essas situações nos seus programas. Para reduzir o impacto das deficiências de operação, verifica-se a necessidade de constante aperfeiçoamento das práticas operacionais.

Considerando as possíveis situações da planta antes da ocorrência do evento, conclui-se que quase em metade dos casos as usinas estavam em operação plena ou parcial.

Os sistemas falhados ou afetados mais citados nos relatórios analisados foram: geradores diesel de emergência $(6,6 \%)$, sistema de refrigeração do reator $(5,6 \%)$ e problemas dos geradores de vapor $(4,7 \%)$. A soma da ocorrência desses três sistemas corresponde a quase $17 \%$ do total investigado.

No grupo dos componentes falhados ou afetados destacam-se as válvulas e seus acionamentos $(15,2 \%)$; dutos e tubos (8,9\%); componentes elétricos $(7,6 \%)$ e, bombas e compressores $(6,9 \%)$. Esses quatro grupos de componentes correspondem a mais de $30 \%$ dos dados analisados.

As causas diretas apontadas possuem baixas ocorrências individuais devido à quantidade de código possíveis, entretanto, se for realizado um agrupamento por afinidade, o conjunto de ocorrências relacionadas à manutenção representam 7,9\% dos eventos, seguido pelas causas relacionadas ao desempenho humano citadas em 7,1\% dos relatórios.

Dentre as possíveis consequências dos eventos, os desligamentos automáticos ou manuais de forma controlada, que podem estar relacionados aos limites das Especificações Técnicas, correspondem a mais de $30 \%$ dos eventos. Se forem considerados no somatório os acionamentos de dispositivos de segurança, esse conjunto de códigos supera $40 \%$ do total. Esses resultados indicam que a cultura existente nas plantas nucleares prioriza a segurança nuclear em detrimento das questões financeiras e, que esse processo de estabelecimento de níveis de defesa precisa ser contínuo.

Sobre as características dos eventos, destacam-se oscilação de potência (11,9 \%), degradações e uma função de segurança $(10,4 \%)$ e degradação no limite de refrigeração do reator $(8,6 \%)$. Agrupando-se as três características de eventos citadas, verifica-se que o conjunto corresponde a mais de $30 \%$ dos eventos.

No grupo de códigos relacionados à natureza das falhas ou erros, destacam-se as falhas simples $(35,7 \%)$, seguidas das falhas ou erros múltiplos em sistemas independentes (17\%). As falhas de causa comum representam 13,2 \% do total, sendo o valor significativamente alto em comparação com os outros tipos de falhas. 
Os resultados para a recuperação da usina demonstram um alto percentual da ação humana, seja através de rotinas e procedimentos previsto $(50,7 \%)$ ou não $(20,9 \%)$. Os eventos relacionados à recuperação por ações automáticas também se mostram significativas, visto que representam 10,3 \% do total.

Finalizando, é importante ressaltar o papel dos profissionais que atuam nas diversas áreas das usinas sobre a segurança operacional das instalações. Dessa forma, verifica-se a importância do treinamento dos profissionais para reduzir os erros humanos e manter as condições de segurança das usinas.

\section{REFERÊNCIAS}

[1] CHEN, L. Safety of Nuclear Energy: Analysis of Events at Commercial Nuclear Power Plants. Tese de Mestrado. ETH Zurich, 2018.

[2] VILLAS BOAS, M. J. Diagnóstico de Classes utilizando Inteligência de Enxames aplicada ao Problema de Identificação de Transientes Nucleares. Dissertação de Mestrado. Fortaleza: Universidade Estadual do Ceará, 2011.

[3] CNEN - Comissão Nacional de Energia Nuclear. Norma NN 1.14 - Relatórios de operação de usinas nucleoelétricas, 2002.

[4] ARAUjO, J. B., VITAL, R. B. N., VITAL, T. M. Nuclear power plant event study based on International Reporting System (IRS). 2019 International Nuclear Atlantic Conference (INAC 2019), Santos, Brazil, 2019.

[5] IAEA - International Atomic Energy Agency. Specific Safety Requirements (SSR-2/2) Safety of Nuclear Power Plants: Commissioning and Operation, 2016.

[6] VITAL, R. B. N., VITAL, T. M. O envelhecimento de cabos de instrumentação e controle em centrais nucleares. Revista Científica do Instituto Federal de São Paulo, v. 19, pp. 37-42, 2018.

[7] IAEA - International Atomic Energy Agency. Reference Data Series $\mathbf{N}^{\circ} \mathbf{2}$ - Nuclear Power Reactors in the world, 2019. 
[8] IAEA - International Atomic Energy Agency. Manual for IRS coding - Joint IAEA/NEA International Reporting System for operating experience - Service Series $\mathbf{N}^{\circ} \mathbf{2 0}, 2011$.

[9] GUIMARÃES, L. A. Metodologia de Gerenciamento da Degradação por Envelhecimento nas Usinas Nucleares de Angra. Dissertação de Mestrado. Rio de Janeiro: CNEN/IEN, 2017.

[10] KANCEV, D., DUCHAC, A., ZERGER, B., MAQUA, M., WATTRELOS, D. Events related to emergency diesel generators in the nuclear industry: Analysis of lessons learned from the operating experience. Progress in Nuclear Energy, v. 75, pp. 192-197, 2014.

[11] BALlESTEROS, A., SANDA, R., MAQUA, M., STEPHAN, J. L. Operational experience data analysis on events related to maintenance at nuclear power plants. Nuclear Science and Engineering, v. 184, pp. 575-583, 2016.

[12] MICHEL, F. Evaluation of operating experience with regard to passive mechanical components - approach and new insights. EUROSAFE 2012, 2012.

[13] ZIEDELIS, S. Summary report on events related to supply of nuclear power plants components. JRC Scientifc and Policy Reports, 2012.

[14] AlvarengA, M. A. B., FRUTUOSO E MELO, P. F., FONSECA, R. A. A critical review of methods and models for evaluating organizational factors in Human Reliability Analysis. Progress in Nuclear Energy, V. 75, pp. 25-41, 2014.

[15] ELETRONUCLEAR. Final Safety Analysis Report - Centra Nuclear Almirante Álvaro Alberto - UNIT 2, rev. 14, 2016.

[16] DELCOURT, J. H. Estudo da confiabilidade do sistema de água gelada de um usina PWR de 4 loops de projeto KWU de 1330 kWe. Dissertação de Mestrado. Rio de Janeiro: Universidade Federal do Rio de Janeiro, 2005. 
[17] ALMEIDA, C. A. B. Oscilações espaciais de Xenônio-135 e fluxo neutrônico. Dissertação de Mestrado. Recife : Universidade Federal de Pernambuco, 2006.

[18] TEIXEIRA, P. I. N. Modelagem de funções críticas de reatores nucleares utilizando Field Programmable Gate Array. Dissertação de Mestrado. Rio de Janeiro: CNEN/IEN, 2016.

[19] COSTA, S. D. N. Proposta de implantação de um programa de monitoração de confiabilidade de sistemas eletrônicos de segurança em usina nuclear. Dissertação de Mestrado. Rio de Janeiro: CNEN/IEN, 2016.

[20] GOMES, M. R. S. Indisponibilidade dos sistema de remoção de calor residual de Angra 1 por Redes Bayesianas considerando falhas dependentes. Dissertação de Mestrado. Rio de Janeiro: Universidade Federal do Rio de Janeiro, 2015. 